\title{
Lockdowns Cause Pollution Dips
}

\author{
Data indicates that the 2020 spring lockdowns in Europe induced \\ measurable drops in gaseous pollutants.
}

By Katherine Wright

$\prod$ he spring of 2020 was a downer. Like many around the world, I was confined to my home, allowed out only for essential business-picking up groceries or medicines-and a daily walk. My husband, infant son, and I were lucky to have the Long Island shoreline just a 15-minute stroll away. During one of our outings in April I noticed something peculiar in the bay. The normally brown haze had lifted, and I was able to clearly see the barrier island that sits a few miles from the coast.

I was not alone in noticing clearer skies during the lockdowns. With cars shuttered in garages and planes grounded in airports, pollution levels plummeted across the world. Speaking last month at the Impact of COVID19 on the Environment online conference, researchers based in the UK presented some initial data showing exactly how pollution levels changed over Europe in the early part of 2020. The researchers hope that the data

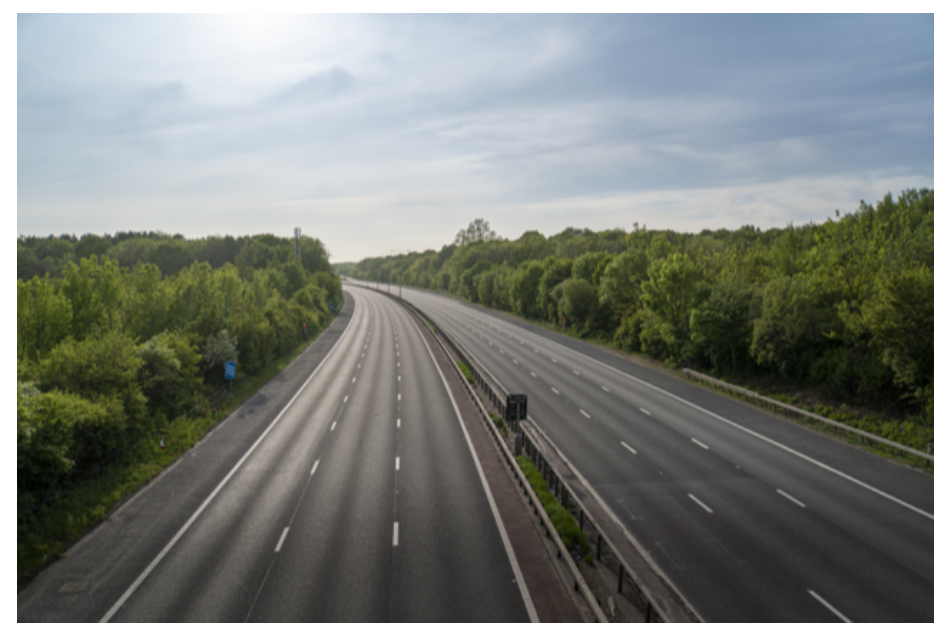

An empty motorway in Kent, UK, during the spring lockdown of 2020.

Credit: smartin69/stock.adobe.com could offer insights into developing future measures to reduce pollutants and greenhouse gases, which, if implemented, could help to alter Earth's warming trajectory.

"Scientists experienced a real-time opportunity [earlier this year] to monitor, observe, and model the response of the environment to perturbations," said Helen Rogers, an atmospheric scientist at the New Model Institute for Technology and Engineering, UK, and one of the organizers of the conference. She says the data may offer "some lessons on how we might press the reset button and mitigate against further climate change."

The conference focused mostly on lockdown-induced changes in nitrogen dioxide $\left(\mathrm{NO}_{2}\right)$ and related oxides. The reason for that is twofold, says Jérôme Barre, a data scientist at the European Centre for Medium-Range Weather Forecasts, UK. First, the sectors impacted by the lockdown are big producers of $\mathrm{NO}_{2}$-around $40 \%$ of the world's $\mathrm{NO}_{2}$ comes from vehicle exhaust and about 30\% from industry. Second, $\mathrm{NO}_{2}$ is short-lived, surviving on the order of just 12 hours. That short lifetime means that $\mathrm{NO}_{2}$ levels should respond more quickly to lockdowns than other gases. "By monitoring $\mathrm{NO}_{2}$ you can see the most drastic effects," Barre said.

Barre presented data from satellites and ground-based observation stations that tracked changes in $\mathrm{NO}_{2}$ over Europe from March 15th to April 30th, a period when most of the continent was locked down. The measurements indicate that the concentration of $\mathrm{NO}_{2}$ fell on average by $30 \%$ over pre-lockdown levels. Data reported by other scientists at the conference shows similar numbers and trends. "COVID has presented the largest perturbation from the baseline atmospheric composition that most of us have seen in our lifetimes," said Lucy Carpenter, an atmospheric chemist at the 
University of York, UK, and one of the conference chairs.

So, what does this perturbation mean for the climate? Chris Smith a climate scientist at the University of Leeds, UK, offered some insights into that question from climate simulations. He and colleagues ran simulations for three main scenarios. The first ignores the lockdown perturbation but assumes that countries meet their Paris Agreement pledges, which allow for a slight global increase in emissions over the next ten years. The second accounts for the lockdown decrease in $\mathrm{NO}_{2}$, as well as in ten other gases, including methane and $\mathrm{CO}_{2}$, which dropped on average by about 4 to $8 \%$. The simulations assume that the lockdown emission changes last for two years and then jump back to pre-lockdown levels. The third scenario assumes a "green recovery" in which emissions continue to drop, with yearly changes matching the lockdown decrease.

The simulations predict that the Paris Agreement baseline leads to a $1.8^{\circ} \mathrm{C}$ increase in Earth's average temperature over pre-industrial levels by 2050. That trajectory is largely unaltered by the two-year lockdown "blip," which Smith showed decreases that baseline temperature by just $1 / 100$ th of a degree. Because of their limited duration, the lockdowns won't have much impact on the climate, Smith said. The green recovery path, however, could significantly alter Earth's warming trend. There, the simulations indicate that Earth's temperature will warm by $1.5^{\circ} \mathrm{C}$ by 2050 .

Smith noted that following the green recovery path would be a challenge for the world, but he's optimistic that it could happen. "Renewable forms of energy are now really, really cheap and getting cheaper," he said. "So I don't think that this path is purely idealistic." That opinion was shared by others at the conference, including Paul Young a climate scientist at Lancaster University, UK. While Young agrees with Smith's conclusion that the lockdowns themselves won't impact climate change, he said that the observed effect on $\mathrm{NO}_{2}$ "has made us realize that the future is not set-there are other possibilities out there."

Katherine Wright is a Senior Editor for Physics. 